\section{COLONIES OF PENICILLIUM NOTATUM AND OTHER MOULDS AS MODELS FOR THE STUDY OF POPULATION GENETICS}

\author{
By Dr. G. PONTECORVO \\ Department of Zoology, \\ AND \\ DR. A. R. GEMMELL \\ Department of Botany, University of Glasgow
}

$\mathrm{E}^{\mathrm{x}}$ XPOSURE of mould colonies to X-rays has long been known to give rise to 'sectorial' mutants. Mutant 'sectors', of course, also arise 'spontaneously' in Fungi as well as in Bacteria. The frequency varies between strains, but is always much smaller than the maximum obtainable by irradiation. Penicillium notatum is no exception, as we have obtained mutant 'sectors' in abundance following irradiation, and occasionally without irradiation. All 'sectors' isolated. gave rise to mutant strains, differing from the original in morphological or metabolic properties. diameter of a colony takes place by multiplication of cells in or near the hyphal tips that form its edge. A nucleus has, thus, a chance to give rise to a patch of mutated mycelium only if it was situated on the growing fringe of the colony at the moment it underwent mutation. But it will also be noticed that whereas 'sectors' of type $a$ taper towards the irradiated edge from which they originate, types $b$ and $c$ originate in a sort of blunt apex. Wo shall see why later.

Before discussing the geometry of 'sectors', let us consider the mode of growth of a mould colony under certain conditions (hard agar and others) which are essential for obtaining regular shapes. Under these conditions, growth of colonies is, as a first approximation, two-dimensional. A hyphal tip or a germinating spore free from competition branches in all directions in a plane so rapidly that it soon forms a circular microcolony ; from this moment the colony grows as a circle, expanding in diameter at a uniform rate so long as there is further medium to occupy. The hyphæ elongate radially, with little intertwining, and branch to fill the increasing space available. The circular expansion of a colony made up of homogeneous mycelium is the resultant of the potentially

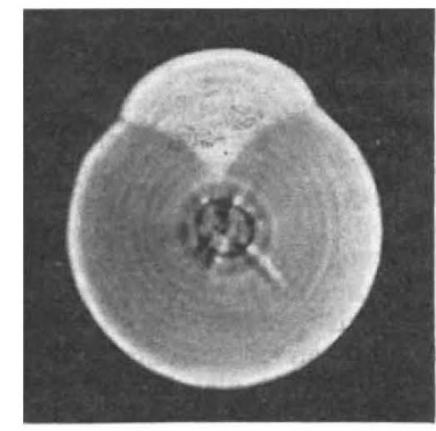

EFig. 1. 'SPONTANEOUS SECTOR' OF Penicillium notatum.

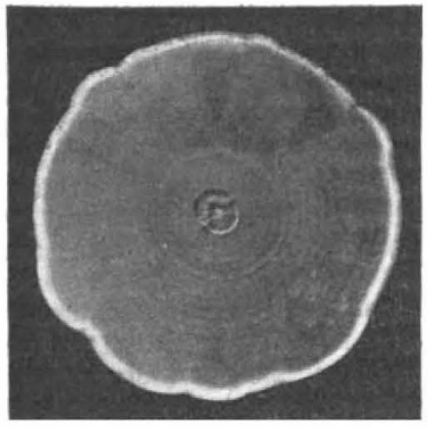

Fig. 2. IrRadiated CONONY ShowING MUTANT 'SEOTORS' OF DIFFERENT SHAPES.

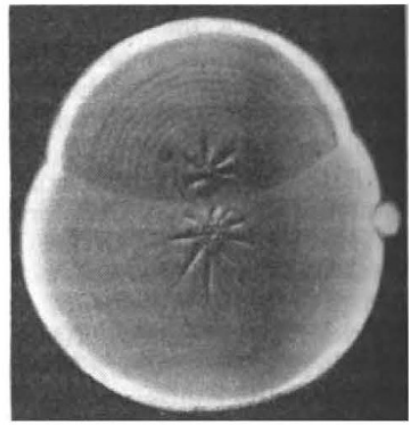

Fig. 8. TWO separate COLONIES WITH DIFFERENT GROWTH-RATES MEET AND COMPETE FOR THE SAME TERRITORY.
The analogy between 'sectoring' in Fungi and somatic mutation, or segregation, in Metazoa and Metaphyta is evident. But the two-dimensional type of growth of mould colonies makes them an unparalleled material for following the fate of a single mutated cell in a growing population of cells. Natural selection and chance can be seen here at work, under measurable conditions, and leave, as it were, a graphical record of their effects.

It is surprising indeed that so little attention has been paid to the strikingly regular shapes of sectors, both 'spontaneous' (Fig. 1) and induced (Fig. 2), and to the significance of these shapes. Even though the term 'sector' is a geometrical misnomer, since the majority have curved, borders or open out at too great an angle, most 'sectors' lend themselves to description in very simple geometrical terms. They may be classified into three elementary types and combinations of them. Fig. 3, $a, b, c$ show diagrammatically these three simple types, and Fig. $3, d$, one of the complex types, a combination of $c$ and $a$. Whereas 'sectors' induced by irradiation may show any one of the three elementary shapes and of their possible combinations, 'spontaneous sectors' in Fungi and Bacteria are almost exclusively of type $a$. It will be noticed (Fig. 3) that X-ray induced sectors have their origin where the edge of the colony was at the time of irradiation. This is simply because the increase in circular expansion of each one of its myriads of hyphal tips, limited by the fact that the territory occupied by one hypha cannot be occupied by another. Hence (1) if a hyphal tip in a large colony were set free from the competition of its neighbours, it would soon produce a roughly semi-circular microcolony bulging out from, and with centre upon, the edge of the larger colony ; (2) competition between hyphæ is extremely severe, and consequently natural selection has plenty of scope for operating.

Let us now suppose that in such a homogeneous colony a hyphal tip suddenly changes its growthrate. If the new growth-rate is lower, usually the mutated hypha will soon be surrounded by branches of its neighbours and prevented from further growth. No trace of the event will be visible. If the new growth-rate is higher, on the other hand, usually the result will be a 'sector' of type $a$ (Fig. 3, a), the boundaries of which are parts of equiangular spirals, with characteristics depending on the ratio of the two growth-rates and the diameter of the mother colony when the changed system arises. A verification of this geometrical explanation can be made by deliberately imitating natural sectors. If a small inoculum is made with a suspension of spores of two strains having different growth-rates, and the spores of slower growth form a big majority, the results are indistinguishable from 'sectors' of type $a$ 
(Fig. 4). It seems that a mutation endowing a hyphal tip with a higher growth-rate is all that is needed to explain the shape of 'spontaneous' and X-ray induced 'sectors' of this type.

The shape of 'sectors' of type $b$ seems, at first, rather mysterious. The straight sides proclaim them to have a growth-rate equal to that of the mother colony. But with an equal growth-rate a hyphal tip. should not be able to produce a 'sector' other than one with vertex at the centre. 'Sectors' of this kind do occur after irradiation, but not frequently. Most type $b$ 'sectors' have vertex away from the centre. Again, the imitation of 'sectors' by mixed inocula has given a clue to a possible mechanism. If a small inoculum is made with a thick suspension of spores of two strains with equal growth-rates, the result is a colony made up of nearly radial sectors of one strain alternating with similar sectors of the other, a sort of rising sun figure (Fig. 5). If, however, the inoculum is large, irregular in shape and contains few spores, some of the sectors open out at great angles. Obviously some initial positional advantage plays a part. The next step is to control this positional advantage by starting two colonies, one of each strain, at different times and near each other (Fig. 6). The results are as those given by the intersection of two systems of concentric circles, expanding at the same rate, but differing in diameter when they first meet. The intersections are hyperbolæ and the angle between the asymptotes depends only on the ratio of the two diameters when the systems first meet (Fig. 3,e). The smaller the diameter of the small colony (or system) in relation to that of the larger one, the smaller becomes this angle and the more nearly do the sides of the resulting 'sector' coincide with the asymptotes and look like straight lines forming a blunt vertex. To explain 'sectors' of type $b$, to which many of those produced by irradiation belong, we have thus only to assume that the mutated hypha either had, immediately after irradiation, a positional advantage over neighbouring hyphæ, or that it enjoyed for a short time a much higher growth-rate. Plausible mechanisms of either type are easily imagined. For example, irradiation may kill a high proportion of hyphal tips and the surviving ones have a greater Lebensraum. Alternatively, differences in metabolism between mutated and non-mutated hyphæ may give one of the former a temporary advantage depending on conditions of the medium temporarily altered by irradiation.

'Sectors' of type $c$ are nothing more than a modification of the preceding case; that is, the mutant has an initial temporary advantage, but a growthrate lower than that of the mother colony. The experimental imitation of 'sectors' of this type needs no comment (Fig. 7).

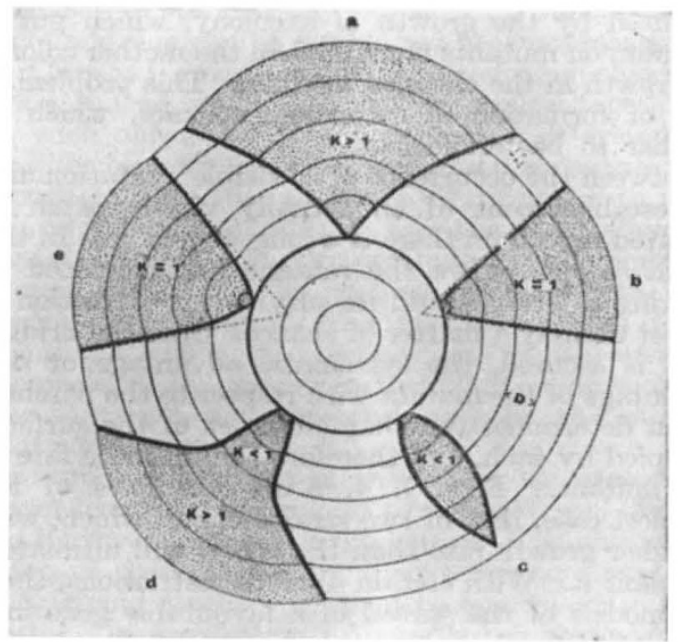

Fig. 3. GEOMETRY OF 'SECTORS' : $a, b, c$, THE THREE SIMPLE TYPES, $d$, ONE OF THE COMPLEX TYPES, $e$, 'SECTOR' OF TYPE $b$ IMITATED BY STARTING TWO COLONIES WITH IQQUAL GROWTH-RATES AT DIFFERENT TTMES. HEAVY CIRCLE INDICATES EDGE OF COLONY
WHEN IRRADIATED. $K$, RATIO OF GROWTH-RATES OF SECTOR AND COLONY ; $D$, INCREASE IN RADIUS PER UNIT TIME.

The types of 'sectors' so far discussed, are those in which mother colony and mutant have growth-rates bearing a fixed ratio to each other over a long period. A number of sectors with shapes interpretable as combinations of those so far described, however, are obviously not of this type. The ratio changes as the colony grows old. This may be due to a special growth habit of the mutant, that is, something detectable even if the mutant is grown by itself, or it may be due to an interaction between mutant and mother colony. Among the mutants we have isolated both types are represented. A simple example of non-reciprocal interaction is that of a mutant which grows more slowly than the normal strain on acid medium and more quickly on alkaline medium. As the $p \mathrm{H}$ of the medium changes (from acid to alkaline) with the growth of a colony, so also does the intersection curve between normal and mutant colonies grown side by side; its final form depends on the time of inoculation of the mutant after the establishment of the normal colony. If very soon (acid medium), the mutant will form a type $c$ 'sector'; if somewhat later (acid medium changing to alkaline), the mutant will form a type $d$ 'sector' ; if still later, the mutant will expand at the expense of the normal.

Cases of reciprocal interaction, namily, antagonism or collaboration, between two or more strains are identifiable and analysable by some such methods. Another problem, in addition, can be investigated along these lines: that of changes in the medium,

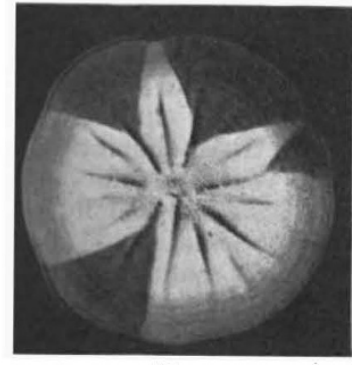

Fig. 4.

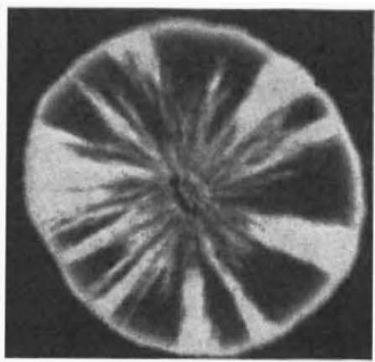

Fig. 5.

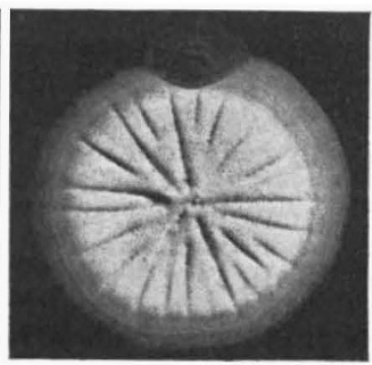

Fig. 6.

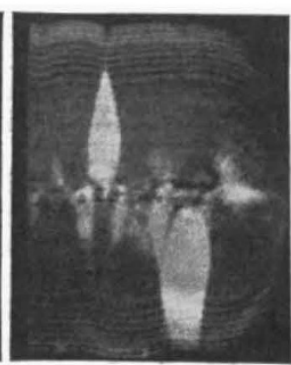

Fig. 7.

Figs. 4-7. IMITATION OF VARIOUS TYPES OF 'SECTORS' BY INOCULA OF TWO STRAINS WITH EQUAL (FIGS. 5, 6) AND DIFFERENT (FIGS. 4, 7) GROWTH-RATES. FIG. 5 GIVES A GRAPHIC MODEL OF THE SEWALI WRIGHT EFFECT. 
produced by the growth of a colony, which put a premium on mutants more fit than the mother colony for growth in the changed medium. This problem is that of formation of secondary colonies, which is familiar to bacteriologists.

Between the occurrence of a 'visible' mutation and the establishment of an actually visible patch of mutated mycelium there is a long way to go. In the initial stages, before the mutant has succeeded in securing a 'bridgehead', its survival or extinction is almost entirely a matter of chance. Once the bridgehead is secured, the systematic advantage or disadvantage of the mutant with respect to the parental strain determines the characteristics of the surfaces occupied by each, and therefore the ultimate fate of the mutant. Figs. 1, 4, 8 are examples of the simplest case, that of two strains one of which, with a higher growth-rate than the other, will ultimately supplant it. With certain obvious restrictions, these are models of the spread of a favourable gene in a population under the stress of natural selection alone. In Fig. 1 the mutant starts as a single individual in a large population; in Fig. 4 the mutant starts as 10 per cent of the initial population; in Fig. 8 two separate populations with different growthrates start at the same time and compete for the same territory : the boundary between them is a circle.

It is, however, as models of the accidental multiplication or elimination of genes, and its dependence on the size of the population, that mutants of moulds cain be most useful. Mixed colonies of strains with equal growth-rates are especially instructive, since in them the effect of natural selection between strains is nil, and that of chance alone stands out. A colony of this type, begun with great numbers of spores of each of two strains, shows (Fig. 5) in its early stages an apparently thorough mixture of both. Later on, the colony becomes made up of clearly distinct sectors each of either one or the other strain. As, with appropriate magnification, the early apparent mixture is seen to result from an alternation of very thin radial strips of each strain, the difference between earlier and later structure is simply a sub. stitution of fewer sectors of great width for an initial very large number of sectors of very small width. Clearly a process of sorting out takes place: sectors which are too small in width either increase in width or disappear. The result is that any small apparently mixed arc in the young colony gives rise, after sufficient growth, to an are in which one strain only is established to the exclusion of the other.

Here is an evident analogy with the Sewall Wright effect of the small size of populations upon the accidental fixation or extinction of genes. In the case of sexually reproducing species, the effect is due to the sampling nature of the reproductive process : accidental fluctuations in the frequency of a gene may, in a small population, reach the irreversible alternatives of either fixation or loss. In mould colonies a similar sampling operates. A hyphal tip in a colony of radius $r$ gives rise, on the average, to $r^{\prime} / r$ hyphal tips when the colony has grown to radius $r^{\prime}$. Accidents of growth, however, allow for fluctuations around this average: one hyphal tip will contribute more to the next 'generation', and another one less and leave no 'progeny'. In a small arc, with a small number of hyphal tips of two strains alternating, these fluctuations will be reversible and preserve the mixed constitution of the are, so long as they do not happen to reach the irreversible state of all hyphæ being of one or the other strain. This will ultimately bring about the segregation of the two strains into 'sectors' of substantial size. To grasp fully the analogy between the Sewall Wright effect in sexually reproducing organisms and our example, one has to substitute a hyphal tip for a gene, and a small arc of the colony for a small population.

There are several possible ways of measuring the intensity of the sampling effects in moulds: unlike the sampling effect of sexual reproduction, its variance may be expected to depend on external conditions.

In conclusion, the remarkable diagrams that mould colonies may be made to produce offer a range of opportunities for the experimental approach to problems as apparently unrelated as morphogenesis and genetics of populations.

Wo wish to thank Miss A. H. Waddell for advice on the mathematical side, and Dr. W. M'Farkane, who carried out the irradiations at the Western Infirmary, Glasgow, for his most helpful contribution to our discussions. A research grant from the Carnegie Trust for the Universities of Scotland has covered part of the cost of materials.

\section{FOUNDATIONS OF ELECTRICAL MEASUREMENT*}

\author{
By DR. L. HARTSHORN \\ National Physical Laboratory
}

$T$ HE last two or three years have seen the publication of a surprising number of papers on the fundamental magnitudes of classical physics. Even more surprising is the divergence of opinion expressed on such well-worn themes as the precise significance of the magnitudes of everyday electricity and magnetism. Well-known expounders of these subjects at our universities have found one another unintelligible, and practising engineers and physicists have frequently been heard to ask if these concepts are really as doubtful as all that after half a century of experience and discussion.

There seems to be fairly general agreement that the working concepts should be defined by reference to the processes by means of which they are measured, but there is no sort of agreement as to what these processes are. It appears that there are many possible processes for each quantity and no agreement as to their relative status. One of the troubles is that the processes described are usually idealized. They have been described as 'theoretical experiments'. It is agreed that they have not actually been performed, but it is asserted that they could be performed in principle; for example, you take two point charges and measure certain distances and forces and so on, operations which appear to the experimentalist of to-day as practically impossible, and in the results of which he would have no confidence whatever.

An outstanding fact, and one which the experimentalist finds very reassuring, is that all the doubt and discussion surrounding these theoretical experiments seem to produce no repercussions on his own real experiments. His values are usually accepted without argument, understood and acted upon with useful results. $\mathrm{He}$ can only conclude that these theoretical operations about which there is so much argument cannot really be the basis of the laboratory work, which remains unaffected. The extent of our

* Based on a lecture to the Measurements Section of the Institution of Electrical Engineers delivered on May 19. 